\title{
Recycled fiber from straw waste: effect of take-up speed and spinneret diameter to linear density and tenacity
}

\author{
Asril S. Soekoco*, Dody Mustafa, Rosmawat, and Jane \\ Politeknik STTT Bandung, Textile Engineering Dept., 40272, Bandung, Indonesia
}

\begin{abstract}
Recycled fiber has a great potential as a solution to meet overgrowing synthetic fiber demand and reduce plastic waste in the same time. In the previous research, bottle cap waste is used as the material of the recycled fiber. The result shows that fiber tenacity of recyled fiber from bottle cap waste has low tenacity, different polymer. This phenomena may have been caused by the present of pigment molecule. This research focused on producing recycled fiber from straw waste which has low pigment content. Straw waste was washed and cleaned before the cutting process. Then, the waste was processed in the experiment melt spinning machine with plunger system and single hole orifice in various diameter, $4 \mathrm{~mm}, 10 \mathrm{~mm}$ and $15 \mathrm{~mm}$. The processing temperature was $140^{\circ} \mathrm{C}$ temperature in three take-up speed, $3.00 \mathrm{~m} /$ minutes, $10.30 \mathrm{~m} / \mathrm{minutes}$ and $19.16 \mathrm{~m} /$ minutes. The diameter and cross section shape of recycled polyethylene fiber were obtained by using electric microscope with software assistance. The linear density of the recycled fiber was analysed by calculating it with denier and the mechanical strength of the fiber was measured in accordance with the ASTM D 3379-75 standard. High take-up speed leads to higher linear density and take-up speed value is proportionally linear with the tenacity of fiber produced. Moreover, the spinneret diameter is proportionally linear with linear density but it is inversely linear with the tenacity of the fiber produced.
\end{abstract}

\section{Introduction}

Currently, plastic is the one of the most important materials in industrial world due to its low production cost, production process and wide range product properties. On the other hand, plastic material needs long time to degrade naturally, and this condition has lead to environmental problems. The demand of plastic product including synthetic fiber climbs up as the growth of clothing demand and populations. Indonesia places as the second rank for mismanaging plastic waste country in the world after China [1]. An effort to fulfil synthetic fiber demand and reduce plastic waste in the same time is one of solution to answer this challenge (Leonas, 2017).

Many research of producing recycled fiber using plastic waste has been developed, and one of the biggest challenge in using waste as material is to yield new the products which have minimum quality deference with the virgin material product. In a previous research, bottle cap waste used as the material of the recycled fiber. The result shows that fiber tenacity of recycled fiber from bottle cap waste has low tenacity, different polymer. This phenomena may have been caused by the present of pigment molecule [2].

This research focuses to produce recycled fiber from waste with straw waste which has low pigment content (see figure 2). Melt spinning method was chosen due to its low process cost and more environmental friendly compared to wet and dry spinning [5]. The melt spinning principle is heating materials to its melting temperature and then extruding the melted materials through small orifice of a spinning nozzle to produce fiber [3]. The fiber produced is then pulled and winded on take-up roll. Fiber take-up velocity can influencing mechanical properties and diameter of the fiber [4]. This research used various spinneret diameter and take-up speed to obtain the optimum fiber property.

\section{Figures and Tables}

\subsection{Material}

Transparent straw waste selected was due to its minimum pigment content. In order to reach homogenous material properties, this research only used transparent straw waste which has $110 \mathrm{~mm}$ length, $3 \mathrm{~mm}$ diameter and 0.24 gram weight (see figure 1). Transparent straw waste washed and cleaned prior to cutting process in $5 \mathrm{~mm}$ length.

Fourier Transform Infra-Red (FTIR) analysis was run on Shimadzu to identify the material polymer. FTIR spectra shows that all these plastic bags waste were polyethylene according to their finger prints. This result confirms to the fact that most of straw in the market is commonly made from polypropylene polymer (see figure 2).

\footnotetext{
* Corresponding author: asril-s@kemenperin.go.id
} 


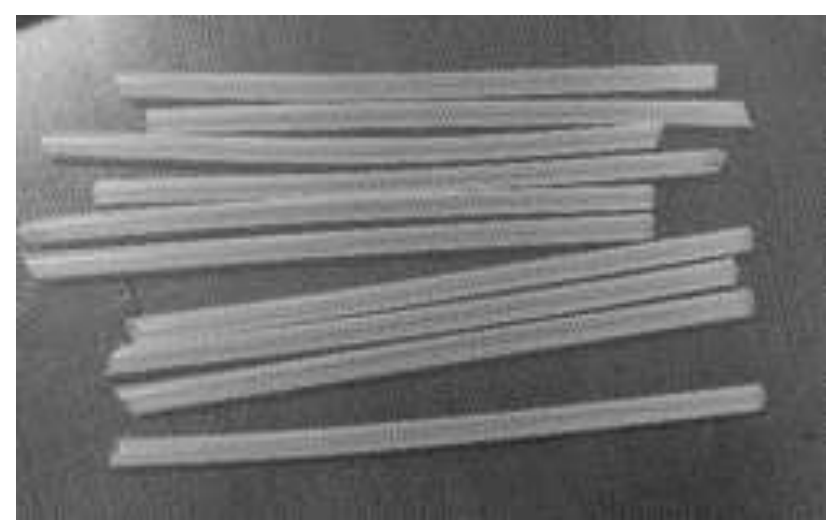

Fig 1. Straw waste

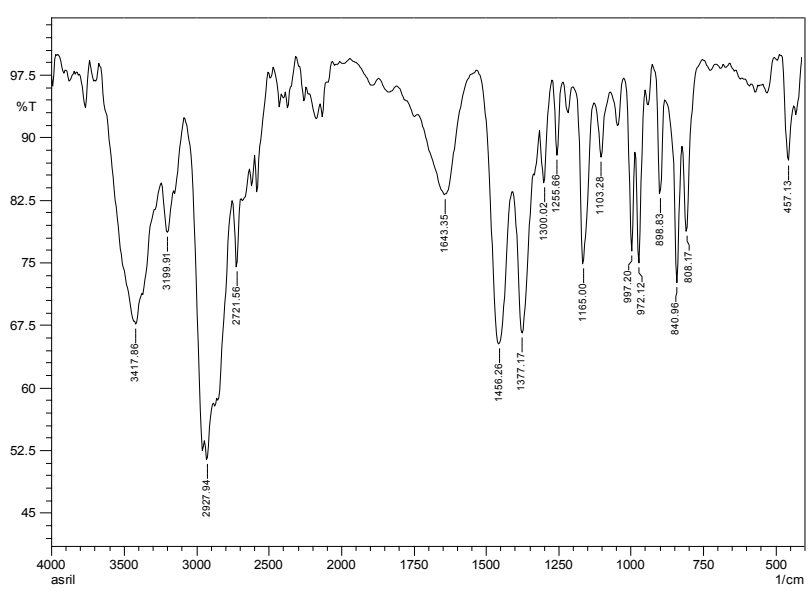

Fig 2. Polypropylene FTIR spectra

\subsection{Melt Spinning Machine}

The melt spinning machine used in this experiment was built using plunger system and utilized by electric motor with speed controller to push molten polymer pass out through spinneret with some adjustment for lab-scale used. The spinnerets have single hole each with $4 \mathrm{~mm}, 10$ $\mathrm{mm}$ and $15 \mathrm{~mm}$ of diameter. The temperature controller was installed in this spinning machine in order to provide precise temperature management. This melt spinning machine has Fotek solid state relay with current load 40 Ampere and barrel with $15 \mathrm{~mm}$ of diameter. The maximum temperature can be reached with this melt spinning machine is $350{ }^{\circ} \mathrm{C}$. The winding unit has various take-up speed, $3.00 \mathrm{~m} /$ minutes, $10.30 \mathrm{~m} /$ minutes and $19,16 \mathrm{~m} /$ minutes and measure using tachometer.

\subsection{Fiber Making}

The fiber production was performed at $130 \pm 2{ }^{\circ} \mathrm{C}$ and plunger movement at $0.25 \mathrm{~m} /$ minutes. After reaching the desired temperature, the heating temperature was maintained for 12 minutes to ensure the uniformity of processing temperature. At the first stage of experiment, 3 variations of take-up speed $(3.00 \mathrm{~m} /$ minutes, $10.30 \mathrm{~m} /$ minutes and $19.16 \mathrm{~m} /$ minutes) applied in $15 \mathrm{~mm}$ of spinneret diameter. At the second stage of the experiment, 3 variations of spinneret ( $4 \mathrm{~mm}, 10 \mathrm{~mm}$ and $15 \mathrm{~mm}$ ) were applied in same take-up speed, $10.3 \mathrm{~m} /$ minutes. All these adjustments were conducted to discover the most suitable setting to produce a fiber with the highest mechanical properties. The molten polymer was than quenched in open air at $24-27^{\circ} \mathrm{C}$ temperature.

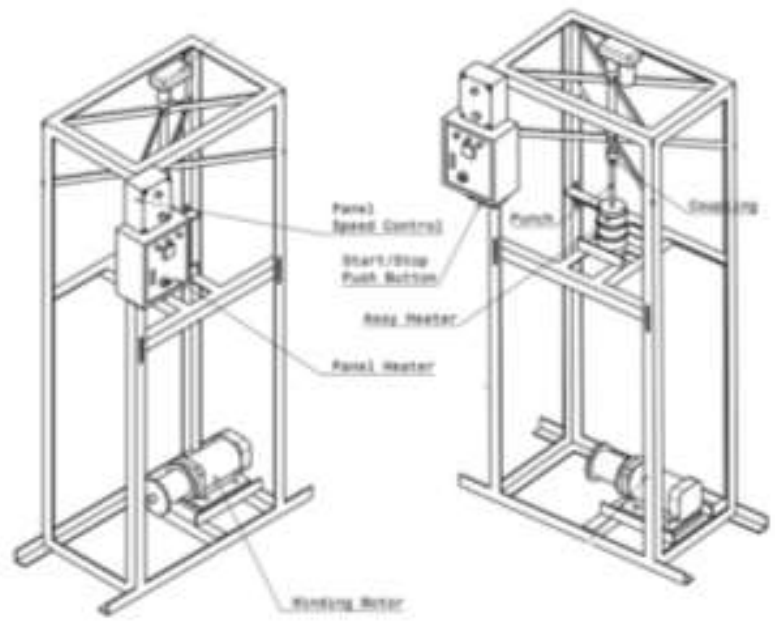

Fig 3. Laboratory scale melt spinning machine

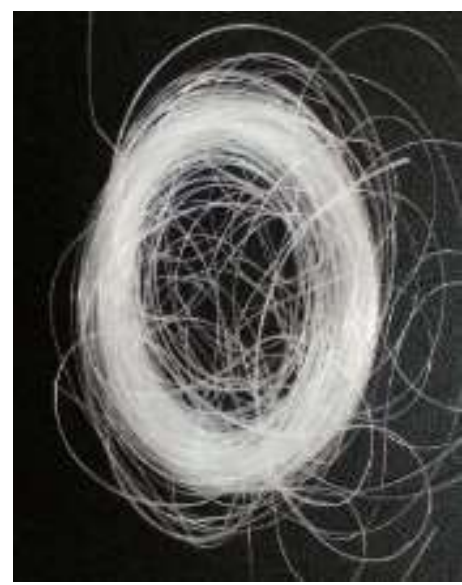

Fig 4. Recycled polypropylene fiber

\subsection{Morphology and Tensile Strength Test}

The mechanical strength of the fibers was measured in accordance with the ASTM standard (D 3379-75) with Textechno Favigraph $100 \mathrm{cN}$ load cell. Fiber diameters were examined using a calibrated optical microscope with software assistance. The fiber examination result unit was pixel, than was converted to micrometre.

\section{Result and Discussion}

\subsection{Fiber Diameter}

The cross section of the fiber was circular as the shape of orifice in spinneret, because the melted polymer was spread by surface stress from take up motion [6]. The fiber diameter of these fibers was varied due to take-up speed and orifice diameter (see figure 5). 


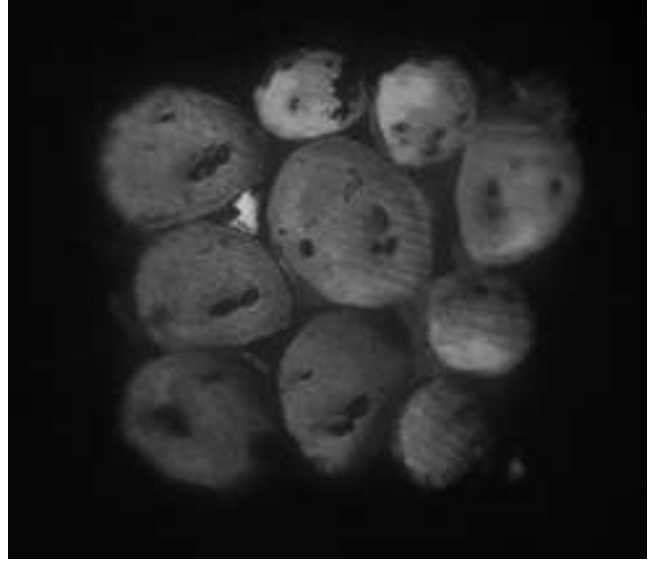

Fig 5. Cross section of recycled polypropylene fiber

\subsection{Linear Density of Fiber}

The linear density of the fiber was influenced by the take up speed and spinneret diameter. High take-up speed and small spinneret diameter lead to fine fiber produced. Comparison of take-up speed to melted polymer throughput is known as draw ratio, which was inversely proportional to linear density, the higher the take-up speed (see figure 6), the higher draw ratio and the finer fibers are produced [7].

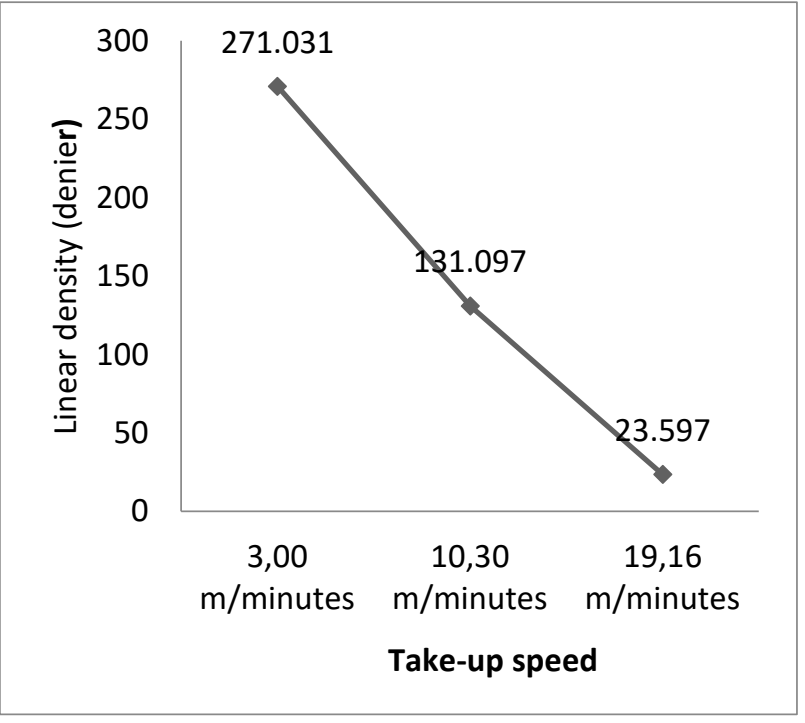

Fig 6. Relationship between take-up speed and linear density

The fiber produced from the highest take-up speed has the smallest linear density and the fiber produced from the lowest take-up speed has the smallest linear density. This phenomena was also influenced by the strain rate which occurred along the spin line. Higher take-up speed leads to higher strain rate. The fiber diameter will decrease as the increment of the distance of fiber from spinneret (see figure 7).

The linear density of the fiber produced was proportionally linear with the spinneret diameter. Bigger spinneret diameter leads to higher molten polymer throughput. Since the take-up speed in this session was fixed in $10.30 \mathrm{~m} / \mathrm{s}$, the highest molten polymer throughput received low strain rate and produced fiber with highest linear density (see figure 8).

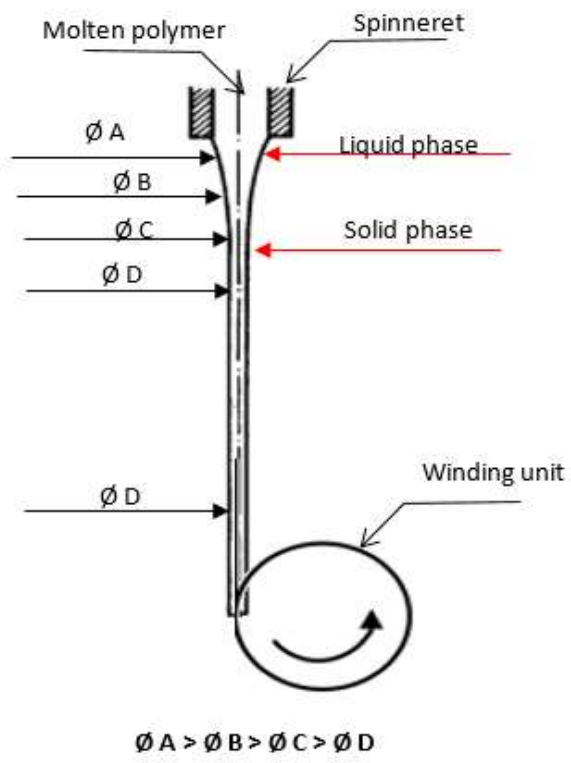

Fig 7. Relationship between take-up speed and fiber finest

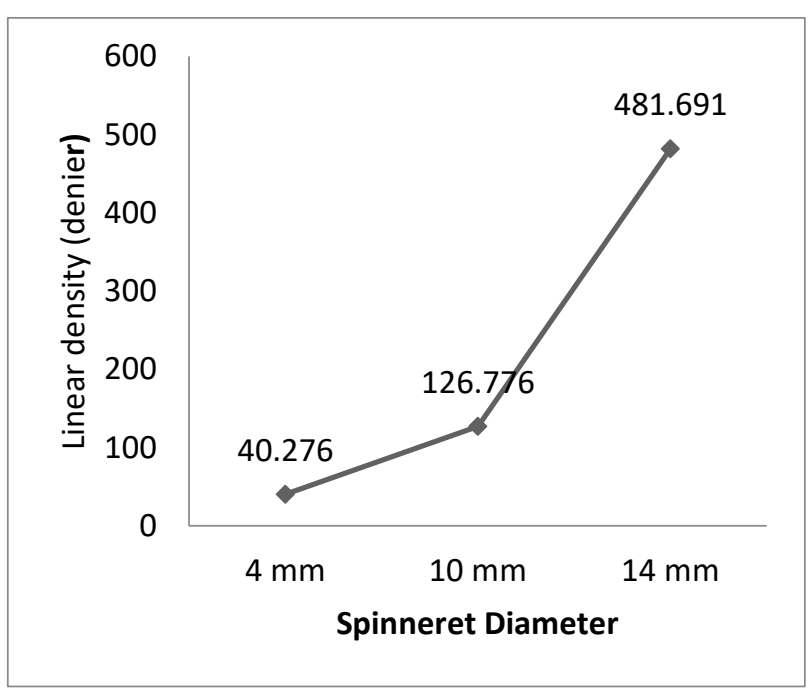

Fig 8. Relationship between spinneret diameter and linear density

\subsection{Tenacity of Fiber}

Tenacity of fiber produced from the highest take-up speed was $9.993 \mathrm{~g} / \mathrm{denier}$ and tenacity of fiber from the lowest take-up speed was $0.666 \mathrm{~g} /$ denier. Take-up speed value is proportionally linear with the tenacity of fiber resulted. This condition is totally different with the second experiment, where spinneret diameter value is inversely linear with the tenacity of fiber resulted. The smallest spinneret diameter produces $3.161 \mathrm{~g} /$ denier of and the biggest spinneret diameter produces $0.787 \mathrm{~g} /$ denier of fiber tenacity (see figure 9 and 10).

The take-up speed is proportional to the draft resulted in the spinning line. Higher draft resulted leads to the increment of polymer molecular orientation degree [7]. 
The increment of polymer molecular orientation improves fiber tenacity (see figure 11).

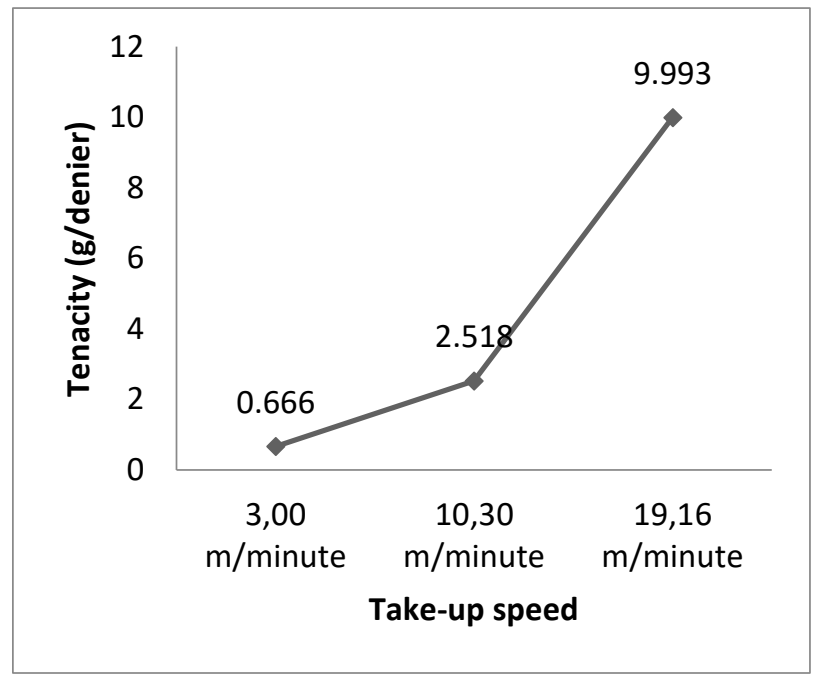

Fig 9. Relationship between take-up speed and tenacity

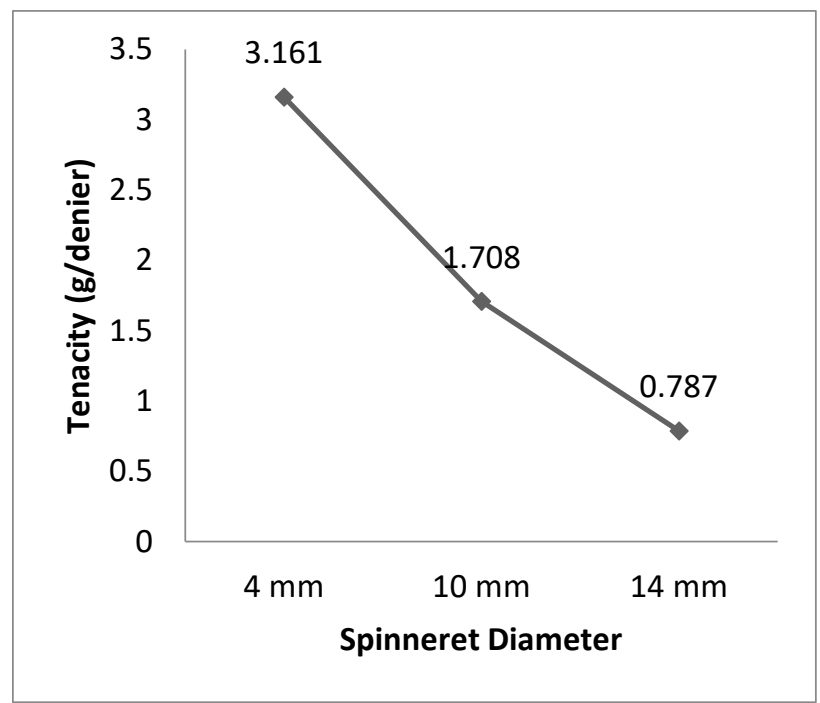

Fig 10. Relationship between spinneret diameter and tenacity

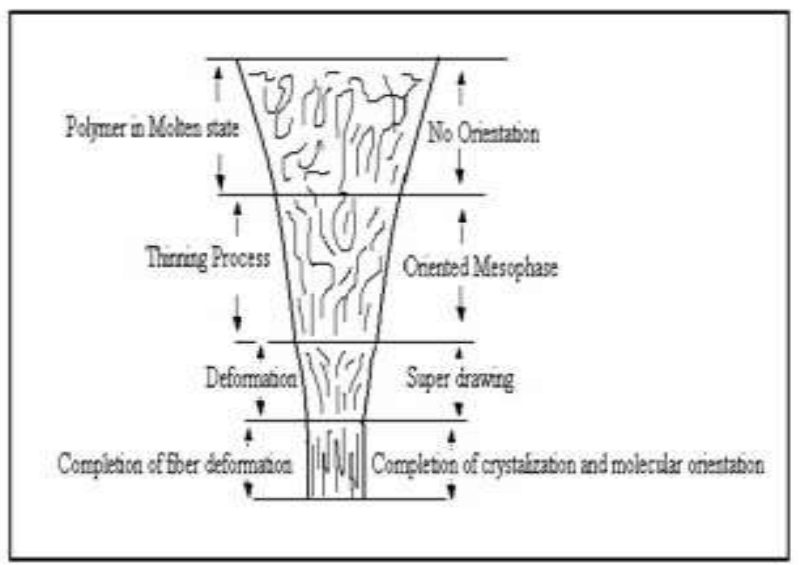

Fig 11. Molecular orientation during drafting

\section{Conclusion}

Transparent straw waste is a potential materials for synthetic fiber by melting spinning method due to its spinability. High take-up speed leads to fine fiber produced and take-up speed value is proportionally linear with the tenacity of fiber resulted. The linear density of the fiber produced from is proportionally linear with the spinneret diameter but inversely linear with the tenacity of fiber resulted.

The authors would like to acknowledge Politeknik STTT Bandung for supporting this research in funding.

\section{References}

1. J. R. Jambeck, M. Perryman, R. Geyer, A. Andrady, C. Wilcox, T. R. Siegler, R. Narayan, K. Lavender. Law Plastic Waste Inputs from Land into the ocean. (2015)

2. A.S. Soekoco, Eco-friendly Fibre from Recycled Polypropylene of Bottle Cap Waste and Lignin, IOP C. S., 105, (2016).

3. F. Luca, I. D, R. Ceccato, Melt Spinning and Drawing of Polyethylene Nanocomposite Fiberwith Organically Modified Hydrotactile, J. Appl. Polym. Sci., (2014)

4. T Nakajima., Advanced Fiber Spinning Technology, (2009).

5. Y. P. Jeon, Simulation of Multifilament Semicrystalline Polymer Fiber Melt-Spinning. (2009).

6. S.J. Eichhorn, W.S. Hearle, M. Jaffe, T. Kikutani. Handbook of Textile Fibre Structure, Woodhead Publishing in Textile New Delhi. (2009)

7. R. R. Hegde, M. G. Kamath, A. Dahiya, Polymer Crystallinity. 